\title{
Intestinal Epithelial Apoptosis Initiates Gross Bowel Necrosis in an Experimental Rat Model of Neonatal Necrotizing Enterocolitis
}

\author{
TAMAS JILLING, JING LU, MICHELE JACKSON, AND MICHAEL S. CAPLAN \\ Department of Pediatrics, Evanston Northwestern Healthcare Research Institute, and Northwestern \\ University, Feinberg School of Medicine, Evanston, Illinois 60201, U.S.A.
}

\begin{abstract}
The histopathology of necrotizing enterocolitis (NEC) is characterized by destruction of the mucosal layer in initial stages and by transmural necrosis of the intestinal wall in advanced stages of the disease. To test the hypothesis that enhanced epithelial apoptosis is an initial event underlying the gross histologic changes, we analyzed epithelial apoptosis and tissue morphology in an animal model of NEC and evaluated the effect of caspase inhibition on the incidence of experimental NEC in this model. Apoptosis was analyzed with terminal deoxynucleotidyltransferase-mediated dUTP-FITC nick end labeling (TUNEL) staining in intestinal sections and by measuring caspase 3 activity from intestinal lysates of neonatal rats subjected to formula feeding and cold/asphyxia stress (FFCAS) and from mother-fed (MF) controls. Morphologic evaluation was based on hematoxylin and eosin staining of intestinal sections. FFCAS resulted in histologic changes consistent with NEC, which were absent from MF animals. FFCAS was also associated with a significantly increased rate of nuclear DNA fragmentation in the small intestinal epithelium compared with MF. Elevated tissue caspase 3 activity confirmed the presence of apoptosis in
\end{abstract}

\section{ABSTRACT}

samples with increased DNA fragmentation. Analysis of the coincidence of morphologic damage and apoptosis in corresponding tissue sections indicated that apoptosis precedes gross morphologic changes in this model. Furthermore, supplementation of formula with 8 boc-aspartyl(OMe)-fluoromethylketone, a pan-caspase inhibitor, significantly reduced the incidences of apoptosis and experimental NEC. These findings indicate that in neonatal rats FFCAS induces epithelial apoptosis that serves as an underlying cause for subsequent gross tissue necrosis. (Pediatr Res 55: 622-629, 2004)
Abbreviations
BAF, 8 boc-aspartyl(OMe)-fluoromethylketone
FFCAS, formula feeding and cold/asphyxia stress
MF, mother-fed
NEC, necrotizing enterocolitis
PAF, platelet-activating factor
TUNEL, terminal deoxynucleotidyltransferase-mediated
dUTP-FITC nick end labeling

NEC occurs in $5-15 \%$ of premature infants born weighing $<1500 \mathrm{~g}$ and remains one of the leading causes of death in these patients (1-3). Nonetheless, the etiology of NEC remains elusive, and no specific treatment or preventive approaches have been successful. Several lines of evidence suggest that neonatal risk factors of prematurity, asphyxia, intestinal ischemia, and formula feeding are all contributing to the occurrence of the disease (4-6). The immaturity of mucosal host defense, inappropriate bacterial colonization profile, and an imbalance of endothelin-dependent vasocon-

Received February 17, 2003; accepted October 17, 2003.

Correspondence: Tamas Jilling, M.D., Department of Pediatrics, Evanston Hospital, Northwestern University Medical School, 2650 Ridge Ave., Evanston, IL 60201, U.S.A.; e-mail: tjilling@northwestern.edu

Supported by Grant 6-FY99-278 from the March of Dimes Foundation, Grant RO1HD37581-01A1 from the National Institutes of Health, and the Jessica Jacobi Golder endowment.

DOI: 10.1203/01.PDR.0000113463.70435.74 striction and nitric oxide-dependent vasodilatation have also been implicated in the disease (7-10). Among the many inflammatory mediators that have been identified as possible culprits in the pathogenesis of NEC, PAF has been shown to play a central role. Elevated serum PAF levels in NEC patients and decreased serum PAF acetylhydrolase (i.e. the PAF degrading enzyme) in premature neonates suggests a role for this molecule in NEC pathogenesis. Initial studies in adult rats using an acute model have shown that exogenous PAF given intravenously results in ischemic bowel necrosis (11) and endotoxin, hypoxia, or tumor necrosis factor (TNF)-induced intestinal injury can be prevented by PAF receptor (PAFR) antagonists $(12,13)$. Furthermore, PAFR antagonists, or recombinant human PAF acetylhydrolase supplementation to formula prevented experimental NEC in a neonatal rat model of the disease. Much less is known regarding the chain of events that occur at the cellular level during the onset of tissue damage. The mechanisms by 
which the aforementioned inflammatory mediators elicit the necrosis of intestinal tissues are poorly understood.

It has been suggested that increased mucosal permeability may be an early event in the pathogenesis of NEC, allowing for the translocation of bacteria, recruitment of polymorphonuclear leukocytes, and consequent tissue destruction $(14,15)$. Because the mucosal barrier is formed by a layer of epithelial cells with tight junctions sealing the gap between the cells, increased epithelial permeability can be caused by loss of tight junctions or damage to the epithelial cells. FAS-mediated apoptosis of cultured $\mathrm{T}_{84}$ colonic adenocarcinoma cells has been shown to cause an increase of transepithelial permeability, but this cultured cell monolayer also exhibited a remarkable ability to restore barrier function in the face of the loss of cells (16). Key factors in NEC pathogenesis, such as hypoxia reoxygenation, $\mathrm{PAF}, \mathrm{TNF}-\alpha$, and endotoxin, have been shown to increase mucosal permeability (17-21). Most of these mediators have been shown to cause apoptosis of intestinal epithelial cells as well (22-24), but the connection between apoptosis and subsequent gross morphologic damage in NEC is yet to be elucidated.

Abundant epithelial apoptosis has been observed in intestinal villi in histologic specimens collected at the time of bowel resection in patients with NEC (25), however it remains unknown whether the observed epithelial apoptosis underlies the gross tissue necrosis, or only coincides with the wide-spread tissue destruction in NEC. Apoptosis of intestinal epithelium occurs during morphogenesis $(26,27)$ and also mediates the physiologic turnover of enterocytes, but increased amounts of apoptosis have been correlated with pathologic conditions in the intestine as well (28-30). It is notable that intestinal epithelial apoptosis is induced by ischemia-reperfusion injury in vivo $(24,28,30)$, and by bacterial invasion (29), peroxynitrite (31), and the inflammatory cytokine TNF- $\alpha$ (29) in vitro. In a surgical model of intestinal ischemia-reperfusion injury, the specific inhibition of apoptosis by a peptide caspase inhibitor reduced histologic damage (28), and the intestine-specific over-expression of $b c l-2$, a prototypical anti-apoptotic member of the $b c l-2$ gene family, resulted in protection from ischemiareperfusion-induced bowel necrosis (24). In the neonatal rat model of NEC, intestinal ischemia-reperfusion injury is likely to play a role, as the cold stress after asphyxia is expected to augment and prolong intestinal ischemia (32-34). Thus, given the suggested underlying role of apoptosis in intestinal ischemiareperfusion injury, we hypothesized that, in the neonatal rat model of NEC, epithelial apoptosis plays an early and underlying role in mucosal injury.

To test this hypothesis, we evaluated apoptosis in the small intestinal epithelium in neonatal rats subjected to FFCAS, and correlated the degree of epithelial apoptotic DNA fragmentation with the histologic damage to the intestinal wall. We have found an increased incidence of abundant epithelial apoptosis in sections of small intestine from neonatal rats that were subjected to FFCAS. We provide novel evidence suggesting that abundant apoptosis precedes gross morphologic damage, and that caspase inhibition significantly reduces the incidence of experimental NEC in this model.

\section{MATERIALS AND METHODS}

Materials. The apoptosis detection system, fluorescein was from Promega (Madison, WI, U.S.A.). Caspase 3 enzymatic activity assay kits were from Biovision (San Diego, CA, U.S.A.), and BAF was from Enzyme Systems Products (Livermore, CA, U.S.A.). All other chemicals were from Sigma Chemical (St. Louis, MO, U.S.A.).

Neonatal rat model. All animal experiments were performed in accordance with the applicable animal welfare regulations and with the approval of the Institutional Animal Care and Use Committee (IACUC\#A3444-01). Experimental NEC was induced in neonatal rats with FFCAS based on a protocol originally described by Barlow and Santulli (35) and further developed in our laboratory (36). Briefly, Pregnant SpragueDawley rats (Harlan Bioproducts for Science, Indianapolis, IN, U.S.A.) were obtained, and after preterm delivery by cesarean section at $\mathrm{d} 21$ of pregnancy, newborns were removed from their mother. Neonatal rats were warmed at $37^{\circ} \mathrm{C}$ and humidified in a newborn incubator. After stabilization, an orogastric feeding tube (silastic catheter, 1.9F) was positioned and feedings instituted using neonatal animal formula (Esbilac, 200 cal $/ \mathrm{kg} / \mathrm{d}$; PetAg, Hampshire, IL, U.S.A.) at $0.1 \mathrm{~mL}$ every $3 \mathrm{~h}$ for $36 \mathrm{~h}$, then $0.2 \mathrm{~mL}$ every $3 \mathrm{~h}$. Animals were weighed daily to document adequate nutritional intake. Hypoxia was accomplished by stressing each rat pup with $100 \% \mathrm{~N}_{2}$ for $50 \mathrm{~s}$, followed by exposure to cold $\left(4^{\circ} \mathrm{C}\right)$ for $10 \mathrm{~min}$ two times daily. Some animals received caspase inhibitors as follows: BAF at a final concentration of $40 \mu \mathrm{M}$ in the formula dissolved from a $20 \mathrm{mM}$ stock in DMSO. Vehicle control consisted of animals receiving DMSO at a 1:500 dilution in formula. The experiment was terminated at 24 or $48 \mathrm{~h}$ and surviving animals were killed by placing them in a chamber filled with $100 \% \mathrm{CO}_{2}$ for $5 \mathrm{~min}$. Half of the intestinal block was fixed in $4 \%$ buffered paraformaldehyde and processed for paraffin embedding, sectioning, and H\&E or TUNEL staining, and the other half was snap frozen in liquid nitrogen, then stored at $-80^{\circ} \mathrm{C}$ for tissue extraction and caspase activity measurement.

TUNEL staining. Formalin-fixed, paraffin-embedded intestinal tissue sections were deparaffinized, treated with proteinase $\mathrm{K}$, and labeled by terminal deoxynucleotide transferase TdT enzyme added along with nucleotide mix including fluorescein-dUTP conjugate. After labeling, preparations were washed and nuclei counter-stained with HOE33258, then slides were mounted with anti-fade reagent (Molecular Probes, Eugene, OR, U.S.A.). In each set of samples, a slide was processed without TdT (negative control) to ensure specificity of positively stained apoptotic bodies. Slides were examined with fluorescence digital imaging or with deconvolution confocal fluorescence imaging using a $20 \times$ objective and the following fluorescence filters: HOE33258 ex: $360 \pm 20$, em: $420 \pm 20$, and FITC ex: $480 \pm 20$, em: $530 \pm 20$. Fluorescence images were then pseudo-colored and overlaid to illustrate the localization of TUNEL positive nuclei relative to all nuclei in the intestine. Specimens were also surveyed by a blinded investigator at a lower magnification using a $5 \times$ objective and were assigned a score in a range of $0-4$. 
Scoring of TUNEL-stained and H\&E-stained slides. TUNEL-stained and H\&E-stained histologic slides were examined by a blinded observer, and were assigned a score on the scale of $0-4$. Slides were prepared such that the H\&E-stained and the corresponding TUNEL-stained slides were from the same set of serial sections from the same block. To illustrate the scoring criteria, Figure 1 summarizes in a schematic the morphologic features (Fig. 1a) and the localization of TUNELpositive nuclei (Fig. $1 a$ and $b$ ) in specimens where apoptosis was observed in the absence of morphologic damage (Fig. 1b) or when apoptosis was associated with histologic damage (Fig. $1 a$ ). Score 0 (both NEC and apoptosis) was assigned to specimens where the villous epithelium was continuous, and only solitaire TUNEL positive nuclei were observed. Specimens with clusters of TUNEL-positive nuclei on the villous tips were assigned an apoptosis score of 1 . Apoptosis score of 2 was assigned to specimens with apoptotic nuclei covering the villous, but crypts were completely spared from apoptosis. Apoptosis score 3 required apoptosis penetrating into the crypts, and apoptosis score 4 was given to specimens with transmural distribution of TUNEL-positive nuclei. The corresponding NEC scores were based on a similar scoring scheme. Intact villi received a score of 0 , sloughing of cells on villous tips was assigned a score of 1 , and mid-villous damage was scored as 2 . NEC score of 3 was assigned when villi were absent, but crypts were still readily detectable; and NEC score of 4 was recorded in cases of complete absence of epithelial structures and transmural necrosis. Scores were always determined based on the highest score observed in a specimen. To evaluate significance
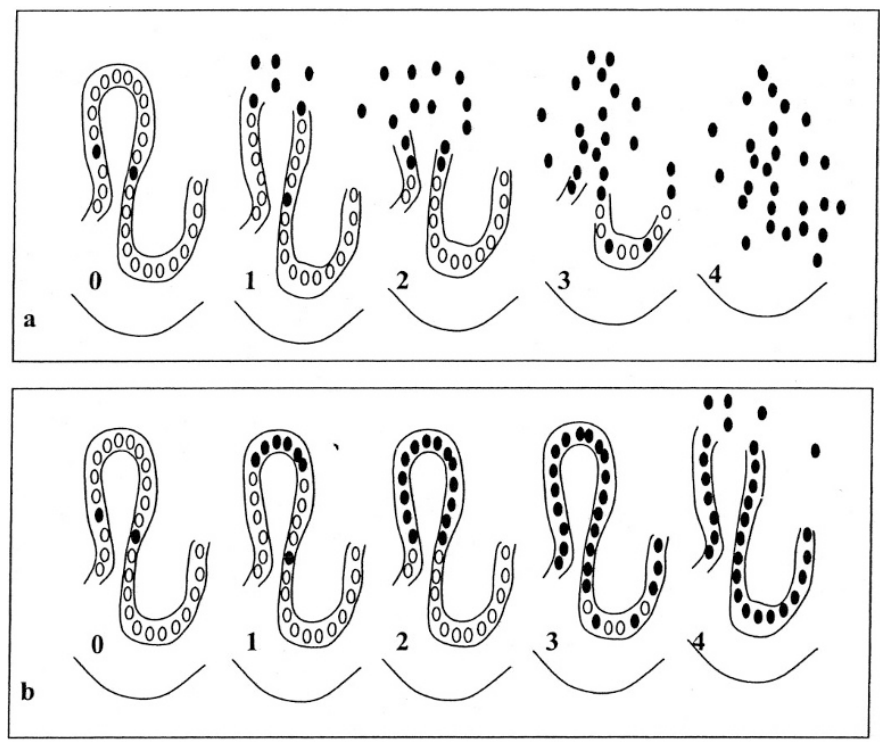

Figure 1. Scoring scheme for the evaluation of apoptosis and necrosis. Based on our initial observations of various occurrences of morphology and apoptosis, we devised a five-grade scale for the quantitative evaluation of apoptosis and tissue necrosis. Apoptosis was evaluated based on TUNEL staining, whereas necrosis was evaluated with $\mathrm{H} \& \mathrm{E}$ histology. For simplicity, all scenarios observed during the course of our studies are summarized on the same schematic. Circles represent nuclei, filled circles represent apoptotic nuclei. Panel $a$ illustrates the simultaneous occurrence of apoptosis and necrosis, and panel $b$ depicts the cases when apoptosis was observed without necrosis. Gross morphologic damage without evident DNA fragmentation was observed only in one specimen.
Table 1. The effect of FFCAS on the incidence of histological NEC and apoptosis

\begin{tabular}{cccc}
\hline & NEC $(-)$ & NEC $(+)$ & NEC $(+) \%$ total \\
\hline MF & & & \\
$24 \mathrm{~h}$ & $32 / 32$ & $0 / 32$ & $0 \%$ \\
$48 \mathrm{~h}$ & $20 / 20$ & $0 / 20$ & $0 \%$ \\
Total & $52 / 52$ & $0 / 52$ & $0 \%$ \\
FFCAS & & & \\
$24 \mathrm{~h}$ & $29 / 41$ & $12 / 41^{*}$ & $35 \%$ \\
$48 \mathrm{~h}$ & $28 / 43$ & $15 / 43^{*}$ & $32 \%$ \\
Total & $57 / 84$ & $27 / 84^{*}$ & $6 \%$ \\
\hline \multicolumn{5}{c}{ Apoptosis $(-)$} & Apoptosis $(+)$ & Apoptosis $(+) \%$ total \\
\hline MF & & & \\
$24 \mathrm{~h}$ & $30 / 32$ & $2 / 32$ & $7 \% \%$ \\
$48 \mathrm{~h}$ & $17 / 20$ & $3 / 20$ & \\
Total & $47 / 52$ & $5 / 52$ & $51 \%$ \\
FFCAS & & & $60 \%$ \\
$24 \mathrm{~h}$ & $20 / 41$ & $21 / 41^{*}$ & $56 \%$ \\
$48 \mathrm{~h}$ & $17 / 43$ & $26 / 43^{*}$ & \\
Total & $37 / 84$ & $47 / 84^{*}$ &
\end{tabular}

Histological NEC and apoptosis scores were determined based on the scoring scheme described in "Materials and Methods" and samples exhibiting scores $>1$ were identified as NEC $(+)$, or apoptosis $(+)$.

$* p<0.001$ vs MF control using $\chi^{2}$ test. See also Figures 1 and 3.

of changes between groups, the total number of observations in each group was divided into two groups based on $\mathrm{H} \& \mathrm{E}$ scores $[\operatorname{NEC}(+)$ and $\operatorname{NEC}(-)]$ and also in two groups based on TUNEL scores [apoptosis $(+)$ and apoptosis $(-)$ ], and the difference between groups was analyzed using $\chi^{2}$ test. Furthermore, the co-existence of apoptosis and necrosis was analyzed, and each specimen received a classification in the following categories: 1$)$ apoptosis $(-) / \mathrm{NEC}(-), 2)$ apoptosis $(+) / \mathrm{NEC}$ $(-)$, and 3) apoptosis $(+) / \operatorname{NEC}(+)$. In the entire set of samples analyzed there was only one specimen that could be categorized as apoptosis $(-) / \mathrm{NEC}(+)$, and thus, this category was ignored in further analyses.

Measurement of caspase activity. Caspase 3 enzymatic activity was analyzed using DEVD-AFC fluorogenic substrate from intestinal tissue lysates using a kit (Biovision, San Diego, $\mathrm{CA}$ ), according to the manufacturer's instructions. Briefly, 10-mm-long segments of neonatal rat intestine were rinsed and homogenized in ice-cold PBS, then lysed in the lysis buffer provided by the manufacturer for $20 \mathrm{~min}$ on ice. The protein

Table 2. The effect of caspase inhibitors on the incidence of intestinal epithelial apoptosis and NEC

\begin{tabular}{lccc}
\hline & Apoptosis $(-)$ & Apoptosis $(+)$ & $\begin{array}{c}\text { Apoptosis }(+) \\
\% \text { Total }\end{array}$ \\
\hline FFCAS (F) & $5 / 25$ & $20 / 25$ & $80 \%$ \\
F + DMSO & $10 / 32$ & $22 / 32$ & $69 \%$ \\
F + BAF & $24 / 32$ & $8 / 32$ & $25 \%$ \\
\hline & Overall significance $p$ & $<0.01$ using $\chi^{2}$ test. \\
\hline & NEC $(-)$ & NEC $(+)$ & NEC $(+) \%$ Total \\
\hline FFCAS (F) & $47 / 88$ & $41 / 88$ & $47 \%$ \\
F + DMSO & $58 / 102$ & $44 / 102$ & $43 \%$ \\
F + BAF & $53 / 69$ & $16 / 69$ & $23 \%$ \\
\hline
\end{tabular}

Overall significance $p<0.01$ using $\chi^{2}$ test. Histological NEC score was determined based on the scoring scheme described in "Materials and Methods" and samples exhibiting scores $>1$ were identified as NEC (+). 

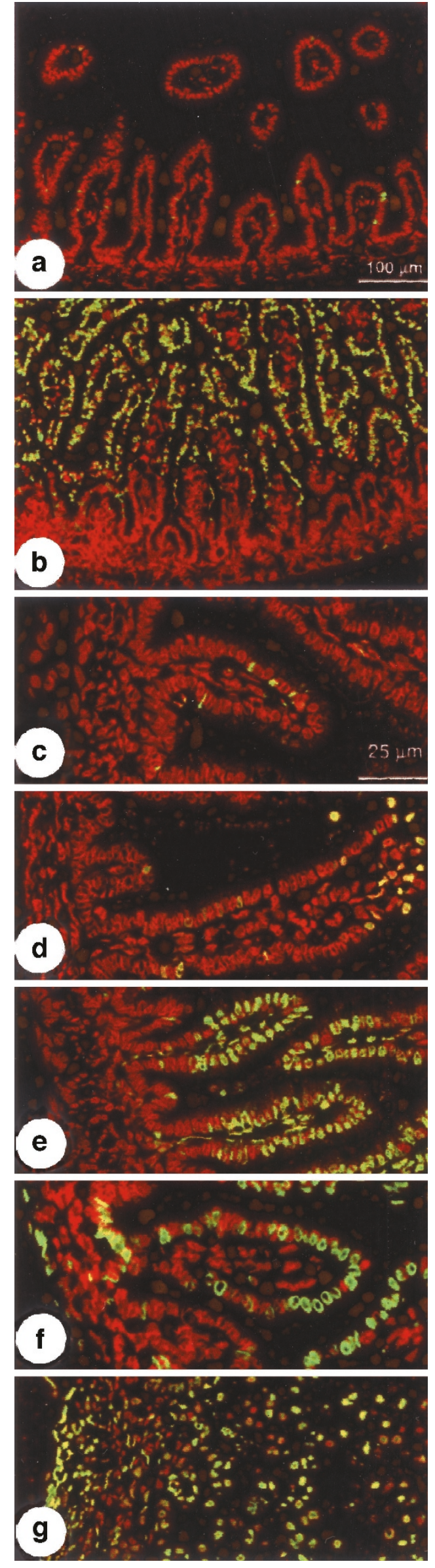

concentrations of the lysates were determined using the BCA colorimetric assay. Subsequently, cell lysates representing equal amounts of protein were mixed with DEVD-AFC, and in parallel reactions with DEVD-AFC and Z-DEVD-FMK (i.e. caspase 3 inhibitory peptide) in incubation buffer $(20 \mathrm{mM}$ HEPES, pH 7.5, 10\% glycerol, 2 mM DTT). After incubation at $37^{\circ} \mathrm{C}$ for $1 \mathrm{~h}$, fluorescence was measured at ex $=380 \mathrm{~nm}$, em $=450 \mathrm{~nm}$. Fluorescence intensity of DEVD-AFC+Z-DEVDFMK-containing reactions was subtracted from the corresponding reactions containing DEVD-AFC only to calculate specific signal.

Statistical analysis. To evaluate changes in caspase 3 activity, specific fluorescence intensities were normalized to the mean intensity of samples representing MF controls, and were expressed as percentage control. Data were analyzed with $t$ test. To evaluate the statistical significance between the incidences of NEC and apoptosis in the various groups, the numbers of observations in each category of each group were entered into contingency tables and analyzed using $\chi^{2}$ test. For data presented in Table 1, $24 \mathrm{~h}$ MF was compared with $24 \mathrm{~h}$ FFCAS in $2 \times 2 \chi^{2}, 48 \mathrm{~h}$ MF was compared with $48 \mathrm{~h}$ FFCAS, and the combined numbers at 24 and $48 \mathrm{~h}$ were also analyzed. For the data shown in Table 2, the numbers of observations were analyzed in $2 \times 3 \chi^{2}$. For both analyses, $\alpha<0.05$ and statistical significance were considered at $p<0.05$.

\section{RESULTS}

Abundant epithelial apoptosis in experimental NEC. We hypothesized that intestinal epithelial apoptosis is an early event in NEC pathogenesis that might underlie subsequent bowel wall necrosis. As shown in Figure 2, we observed a dramatic increase in the frequency of nuclei exhibiting DNA fragmentation in the epithelial layer of FFCAS animals (Fig. $2 b$ ), compared with MF controls (Fig. 2a). To assess the relationship between DNA fragmentation in enterocytes and tissue necrosis in the intestine, we developed a scoring scheme to quantitatively evaluate these alterations (see "Materials and Methods"). Figure 2, $c-g$, depicts examples of TUNEL-stained sections of rat small bowel, illustrating the basis of apoptosis scoring scheme. Figure 2, $b, e$, and $f$, are typical examples of tissue regions where intense TUNEL-positive staining is observed in the top half of the villi (apoptosis score $=2$ or 3 ), but there were no noticeable alterations in morphology based on the morphology deciphered from the nuclear counter-stain (red) and the scoring of corresponding H\&E-stained speci-

Figure 2. Images illustrating the scoring scheme for apoptosis. Shown are TUNEL stained $(a-g)$ sections of neonatal rat small bowel specimens from MF control animals ( $a$ and $c$ ) and FFCAS-treated neonatal rats ( $b$ and $d-g$ ) exhibiting various degrees of DNA fragmentation. Images are results of pseudo-colored overlays of corresponding TUNEL-FITC (shown in green) and Hoechst nuclear counter stain (shown in red). Panel $a$ shows a representative low magnification image of solitary apoptotic nuclei in a MF control, whereas panel $b$ illustrates widespread apoptosis in the epithelial layer at low magnification in a FFCAS-treated neonatal rat. Panels $c-g$ depict various examples of apoptosis scores illustrated at a higher magnification: Panel $c$, apoptosis grade $=0 ; d$, apoptosis grade $=1 ; e$, apoptosis grade $=2 ; f$, apoptosis grade $=3, g$, apoptosis grade $=4$. 
mens. To obtain a corresponding unbiased score regarding morphologic damage, slides cut from the same block were stained with $\mathrm{H} \& \mathrm{E}$ and scored based on the criteria outlined in methods. Figure 3 illustrates examples of each score.

Elevated caspase activity in intestinal samples exhibiting enhanced TUNEL-positive staining. Given that TUNEL staining has been known to produce false-positive reactions occasionally, we measured caspase 3 activity from a selected set of samples. We selected lysates of intestinal segments from FFCAS-treated animals that corresponded to histologic specimens where we observed apoptosis score $>1$, but normal histology (NEC score $=0$ or 1 ), and compared caspase activity in these samples to caspase activity in intestinal segments from MF animals that exhibited only baseline levels of DNA fragmentation (score 0 ). We have found significantly elevated caspase 3 activity from tissue lysates (Fig. 4) of FFCAS animals that had apoptosis scores $>1$, confirming that the TUNEL-positive staining represented apoptosis.

FFCAS results in a significantly increased incidence of NEC and epithelial apoptosis. Figure 4 summarizes our data obtained by determining H\&E scores and corresponding TUNEL scores in a large number of specimens. Figure $5 a$ illustrates the distribution of NEC scores at $24 \mathrm{~h}$ and $48 \mathrm{~h}$ in MF and in FFCAS animals, and corresponding apoptosis scores are shown in Figure $5 b$. Grades $>1$ in both NEC scores and in apoptosis scores were clearly associated with FFCAS in a time-dependent manner (i.e. scores $>1$ increased from 24 to $48 \mathrm{~h}$ in the FFCAS group). To statistically evaluate these changes, we designated NEC grades $>1$ as pathologic, or NEC $(+)$, and apoptosis scores $>1$ as pathologically increased apoptosis, or apoptosis $(+)$. Based on these criteria, we assigned each sample into NEC $(+)$ or NEC $(-)$ and an apoptosis $(+)$ and apoptosis $(-)$ category. By examining the distribution of these categories among MF and FFCAS neonatal rats we found that there was a highly significant $(p<0.001)$ increase in the rate of both NEC (+) and apoptosis $(+)$ in the FFCAS group (Table 1).

Increased apoptosis precedes histologic NEC. To determine whether accelerated apoptosis can be viewed as a potential initiating event in NEC, we determined whether apoptosis precedes NEC in this model. Because the neonatal rat model of NEC does not allow the sampling of tissues at various time points in the same animal, we designed an indirect method to evaluate the relative timing of enhanced apoptosis and NEC. We hypothesized that if apoptosis precedes NEC, we should identify apoptosis $(+)$ in several samples before histologic evidence of NEC has occurred, and in every specimen demonstrating NEC (+), apoptosis should be present. We tested the coincidence of these two designations for each sample (Fig. 6) and found that the data perfectly fit our hypothesis and every specimen exhibiting NEC $(+)$ was also apoptosis $(+)$, and, in approximately one third of specimens from FFCAS-treated neonatal rats, we found evidence of pathologically increased apoptosis but no signs of pathologic histology. These findings are consistent with our hypothesis that enhanced apoptosis precedes, and perhaps underlies, gross histologic alterations.

Supplementation of formula with BAF, a pan-caspase inhibitor attenuates the incidence of histologic NEC. Because
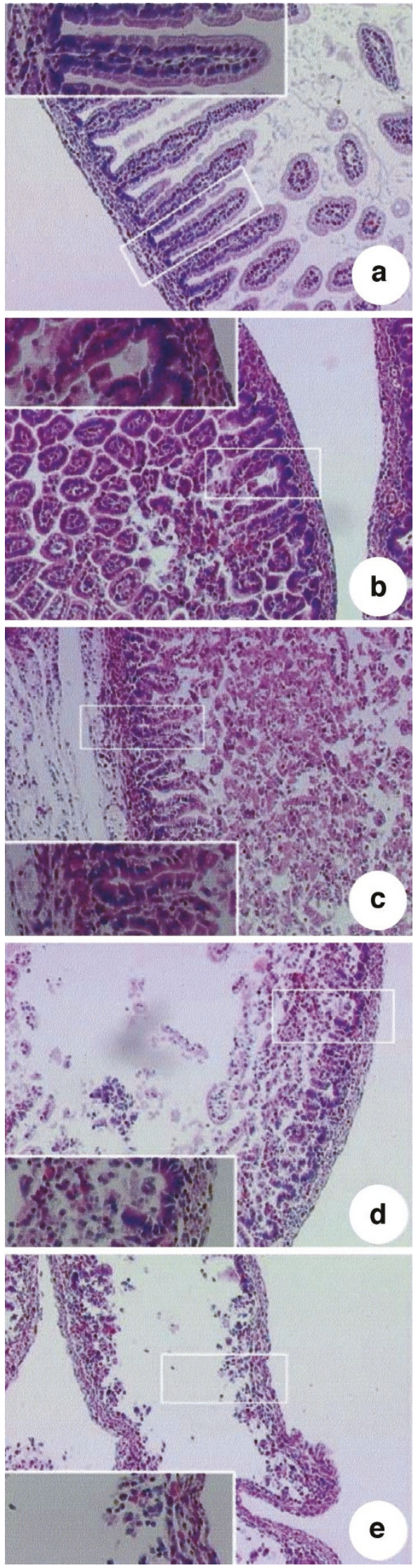

Figure 3. Examples of the various grades of morphologic damage as observed in H\&E-stained specimens. Panel $a$, intact morphology, grade $0 ; b$, sloughing of villas tips, grade $1 ; c$, mid-villas necrosis, grade $2 ; d$, loss of villi, grade $3 ; e$, complete destruction of the mucosa, grade 4 . 


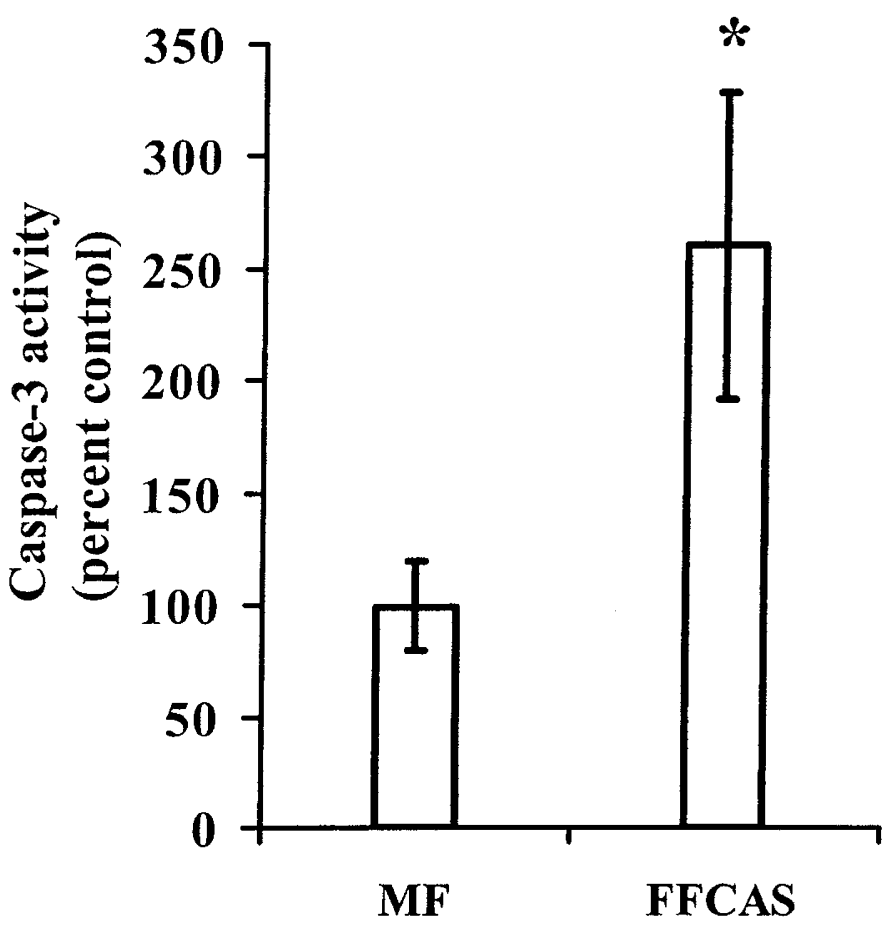

Figure 4. Elevated caspase 3 activity in intestines of FFCAS-treated neonatal rats. Caspase 3 activity was determined from intestinal lysates of MF animals exhibiting normal intestinal morphology and no pathologic DNA fragmentation and from intestinal lysates of FFCAS-treated animals exhibiting normal intestinal morphology, but pathologic DNA fragmentation (grade $>1$ ) as described in "Materials and Methods." *Statistically significant difference at $p<0.05$ ( $t$ test).

our analysis of NEC and apoptosis scores suggested that enhanced apoptosis precedes gross histologic changes, we wanted to evaluate whether apoptosis underlies subsequent morphologic damage. To address this issue we used BAF, a pan-caspase inhibitor supplemented in formula to inhibit intestinal apoptosis, and compared the incidence of apoptosis $(+)$ and NEC $(+)$ in the presence and absence of caspase inhibition. As expected, inclusion of BAF into the formula significantly reduced the rate of pathologic DNA fragmentation in FFCAS-treated neonatal rats (Table 2, top). More importantly, BAF also decreased the rate of histologic NEC in these animals, indicating that inhibition of apoptosis prevented experimental NEC.

\section{DISCUSSION}

NEC is one of the leading causes of death for premature infants weighting $<1500$ g. Despite intense research efforts, only two risk factors - prematurity and formula feeding - have been unambiguously identified for this disease. Additional factors that have been suggested to contribute to the etiology are hypoxia, hypotension, undeveloped regulation of intestinal circulation (7), and intestinal colonization of pathologic bacteria (37). Feeding with mother's milk has been shown to provide some protection against NEC, albeit by unknown mechanism(s) (38). Analysis of serum and fecal samples from affected infants, surgical specimens obtained during bowel resection, and experimental data from animal models of NEC

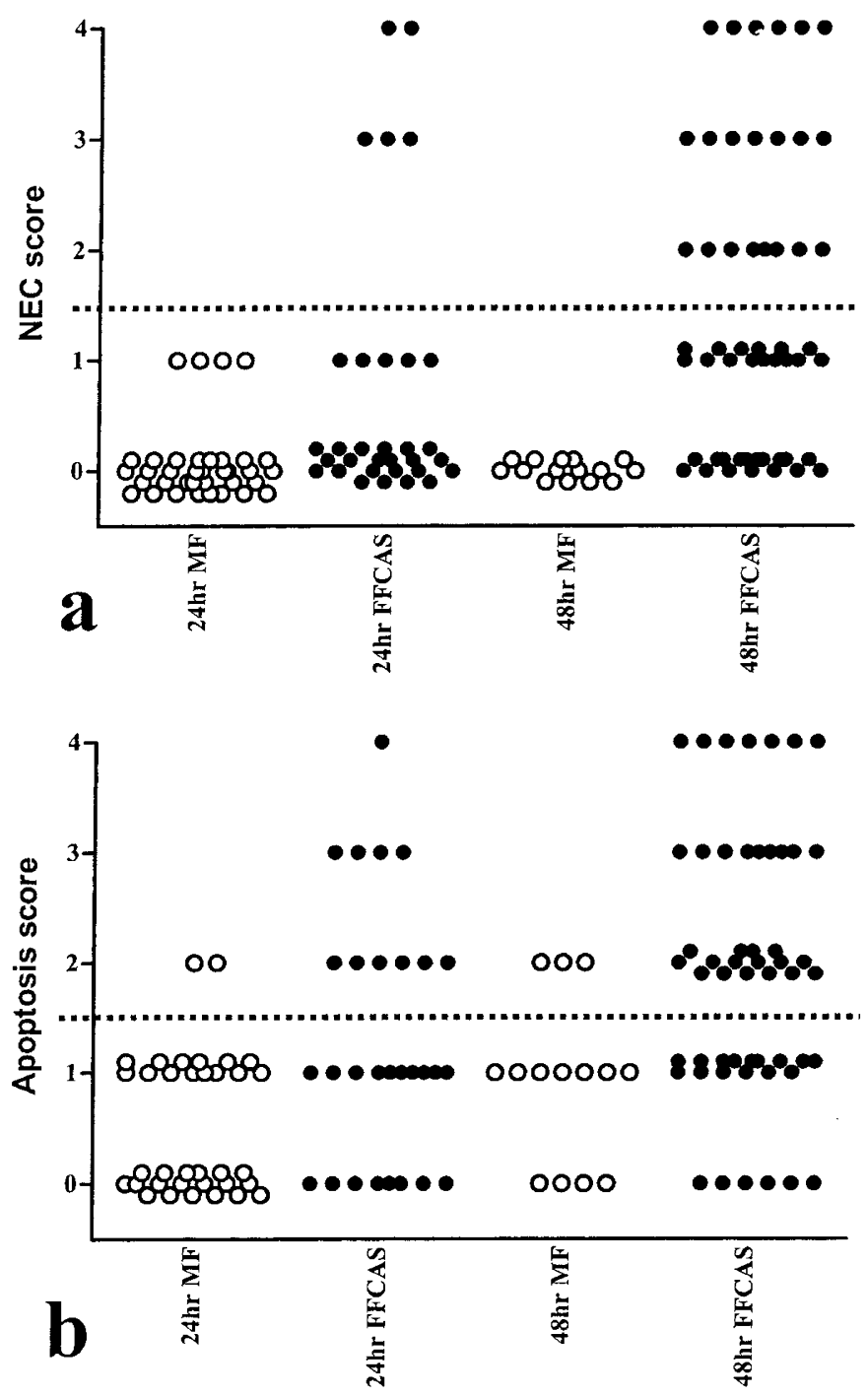

Figure 5. Summary of NEC and apoptosis grade distributions in MF and FFCAS-treated neonatal rats at 24 and $48 \mathrm{~h}$ of life. Panel $a$ depicts the distribution of NEC scores and panel $b$ illustrates the distribution of apoptosis scores. Dashed line indicates the threshold of normal vs pathologic between grade 1 and 2 in both the apoptosis and NEC scores. This threshold was chosen because grades $>1$ were clearly associated with FFCAS.

have identified PAF, TNF- $\alpha$, nitric oxide, and lipopolysaccharides as potential key mediators of the disease $(11,25,39,40)$. Increase of mucosal permeability has been reported in animal models of NEC, and it was suggested that this increased mucosal permeability, the ensuing bacterial translocation to the submucosa, and the activation of the secondary inflammatory cascade are key steps in the final collapse of mucosal integrity (41). The permeability barrier between the lumen of the gut and the submucosa is formed by a layer of epithelial cells and a seal composed by tight junctions between the epithelial cells. The two obvious mechanisms that may lead to a collapse of this barrier function are the loss of tight junctions and/or the loss of cells. Although our study was not designed to specifically evaluate the role of apoptosis in mucosal barrier function, our data are consistent with the notion that a loss of cells due to pathologically increased rates of epithelial apoptosis might significantly contribute to the increased mucosal permeability 


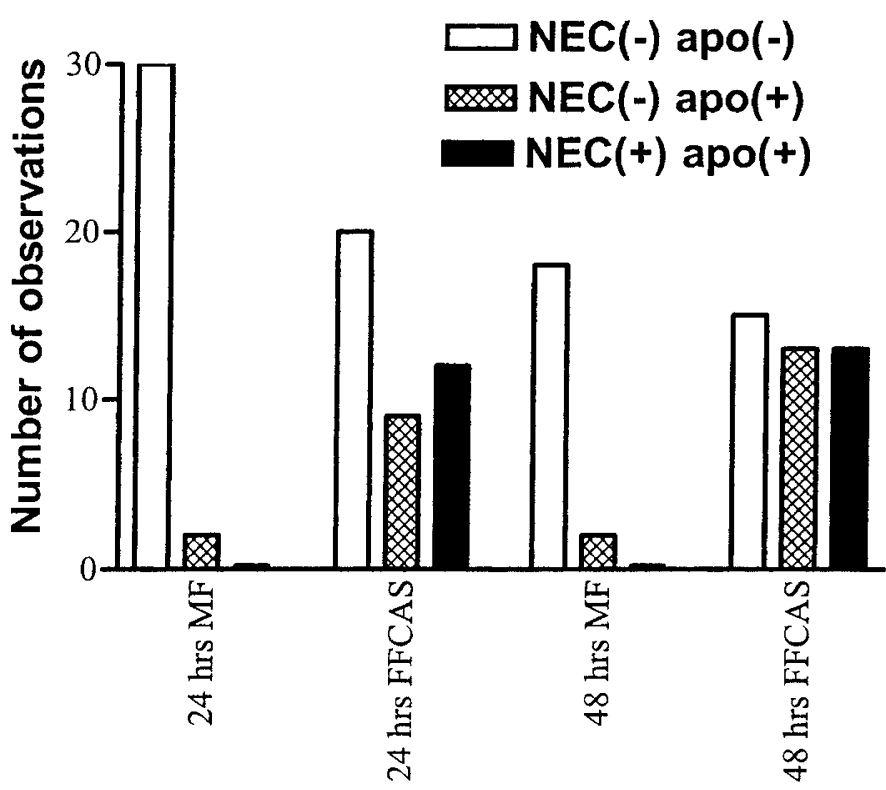

Figure 6. Analysis of the coincidence of morphologic damage and DNA fragmentation in FFCAS-treated neonatal rats. Observations of morphologic changes and DNA fragmentation were divided into three groups: 1) intact morphology with no pathologic DNA fragmentation (clear bar), 2) intact morphology with pathologic DNA fragmentation (shaded bar), and 3) pathologic morphology with pathologic DNA fragmentation (solid bar). A significant portion of samples (one third at $48 \mathrm{~h}$ ) exhibited increased DNA fragmentation without gross morphologic damage.

in NEC. These findings are in agreement with results from human bowel specimens that were obtained from surgical treatment of NEC that revealed the presence of increased DNA fragmentation in the intestinal epithelium in NEC (25).

At the molecular level, apoptosis is regulated by a number of converging signaling cascades that influence each other at various checkpoints along their progression. In most cases, apoptosis involves the activation of select caspases that are characteristic of the signal event initiating apoptosis (42). Nevertheless, DNA fragmentation can occur in the absence of detectable caspase activation (43) or even in necrosis (44). Therefore, we have found it important to measure caspase activity from intestinal lysates as a confirmation that the detected TUNEL staining correlates with enhanced apoptotic activity. The results show that FFCAS-induced increased DNA fragmentation, as detected with TUNEL staining, correlates with elevated tissue caspase 3 activity. Furthermore, BAF, a pan-caspase inhibitor, significantly reduced the incidence of pathologically elevated level of DNA fragmentation. These findings indicate that FFCAS elicits DNA fragmentation in intestinal epithelial cells of neonatal rats via caspase activation, and that the observed TUNEL staining is indicative of apoptosis.

Apoptosis is an underlying mechanism for a number of physiologic processes, including organ development, the elimination of autoreactive lymphocytes, and the resolution of inflammation. Defective regulation of apoptosis has been considered in a number of pathologic conditions (45). For example, aberrant expression of anti-apoptotic gene products has been implicated in malignancies (46) and defective regulation of apoptosis might be the underlying mechanism in the pathology of autoimmune diseases (47). Ischemia reperfusion injury causes apoptosis in the epithelium of the small intestine (24, 30 ) in a manner that can be prevented by the forced overexpression of the anti-apoptotic gene $b c l-2$ (24). It is unknown whether this increased rate of apoptosis is an underlying mechanism of tissue damage, or if it occurs as a form of necessary demolition before tissue restoration. To investigate this issue in our model, we asked the question whether apoptosis precedes gross tissue necrosis. In the neonatal model of NEC it is impossible to follow the course of events within the same animal, therefore, we had to rely on an indirect method to investigate this issue. We postulated the following: If apoptosis precedes NEC, every section with histologic damage has to exhibit increased apoptosis, and there has to be a number of specimens where apoptosis is observed even in the absence of detectable tissue necrosis. Abundant TUNEL-positive staining was observed in every tissue section that corresponded to the histologic evidence of NEC in H\&E-stained serial sections. More significantly, in many cases we observed TUNEL positivity in sections where the corresponding H\&E-stained serial sections lacked evident signs of tissue necrosis. These data indicate that epithelial apoptosis occurs before gross tissue necrosis. To determine whether there is a cause and consequence relationship between epithelial apoptosis and gross tissue necrosis we used a pan-caspase inhibitor, BAF, to block apoptosis and to evaluate whether blocking apoptosis can prevent gross histologic damage in our model. Including BAF in the formula significantly reduced the incidence of apoptosis and NEC in neonatal rats. These data strongly suggest that an increase in the rate of epithelial apoptosis does not only precede the subsequent gross intestinal wall necrosis, but that it is an underlying cause. Further studies will be required to determine the reason for the acceleration of epithelial apoptosis in this model, to investigate the role of apoptosis in increased mucosal permeability, and to investigate the mechanism that leads to massive tissue destruction on the basis of the epithelial damage.

\section{CONCLUSION}

In summary, our studies indicate that abundant apoptosis of the intestinal epithelium precedes widespread tissue damage in an experimental model of neonatal NEC. Given that caspase inhibition protects neonatal rats from FFCAS-induced intestinal injury, apoptosis appears to be an underlying cause of subsequent gross mucosal damage in this model. Although it is unclear whether caspase inhibition could be used safely in the newborn as a potential therapeutic strategy, a better understanding of signaling and execution mechanisms that lead to programmed cell death in enterocytes might provide the basis for the design of future therapeutic and/or preventative strategies for NEC.

\section{REFERENCES}

1. Kliegman RM, Fanaroff AA 1984 Necrotizing enterocolitis. N Engl J Med 310:10931103

2. Ryder RW, Shelton JD, Guinan ME 1980 Necrotizing enterocolitis: a prospective multicenter investigation. Am J Epidemiol 112:113-123 
3. Uauy RD, Fanaroff AA, Korones SB, Phillips EA, Phillips JB, Wright LL 1991 Necrotizing enterocolitis in very low birth weight infants: biodemographic and clinical correlates. National Institute of Child Health and Human Development Neonatal Research Network. J Pediatr 119:630-638

4. Nowicki PT 1998 Effects of sustained flow reduction on postnatal intestinal circulation. Am J Physiol 275:G758-G768

5. Caplan MS, MacKendrick W 1994 Inflammatory mediators and intestinal injury. Clin Perinatol 21:235-246

6. Kliegman RM 1990 Models of the pathogenesis of necrotizing enterocolitis. J Pediatr 117(1 Pt 2):56-513

7. Reber KM, Nankervis CA, Nowicki PT 2002 Newborn intestinal circulation. Physiology and pathophysiology. Clin Perinatol 29:23-39

8. Butel MJ, Roland N, Hibert A, Popot F, Favre A, Tessedre AC, Bensaada M, Rimbault A, Szylit O 1998 Clostridial pathogenicity in experimental necrotising enterocolitis in gnotobiotic quails and protective role of bifidobacteria. J Med Microbiol 47:391-399

9. Furlano RI, Walker WA 1998 Immaturity of gastrointestinal host defense in newborns and gastrointestinal disease states. Adv Pediatr 45:201-222

10. Caplan MS, Miller-Catchpole R, Kaup S, Russell T, Lickerman M, Amer M, Xiao Y, Thomson R, Jr 1999 Bifidobacterial supplementation reduces the incidence of necrotizing enterocolitis in a neonatal rat model. Gastroenterology 117:577-583

11. Gonzalez-Crussi F, Hsueh W 1983 Experimental model of ischemic bowel necrosis The role of platelet-activating factor and endotoxin. Am J Pathol 112:127-135

12. Hsueh W, Gonzalez-Crussi F, Arroyave JL, Anderson RC, Lee ML, Houlihan WJ 1986 Platelet activating factor-induced ischemic bowel necrosis: the effect of PAF antagonists. Eur J Pharmacol 123:79-83

13. Sun XM, Hsueh W 1988 Bowel necrosis induced by tumor necrosis factor in rats is mediated by platelet-activating factor. J Clin Invest 81:1328-1331

14. Deitch EA 1994 Role of bacterial translocation in necrotizing enterocolitis. Acta Paediatr Suppl 396:33-36

15. Piena M, Albers MJ, Van Haard PM, Gischler S, Tibboel D 1998 Introduction of enteral feeding in neonates on extracorporeal membrane oxygenation after evaluation of intestinal permeability changes. J Pediatr Surg 33:30-34

16. Abreu MT, Palladino AA, Arnold ET, Kwon RS, McRoberts JA 2000 Modulation of barrier function during Fas-mediated apoptosis in human intestinal epithelial cells. Gastroenterology 119:1524-1536

17. Mishima S, Xu D, Deitch EA 1999 Increase in endotoxin-induced mucosal permeability is related to increased nitric oxide synthase activity using the Ussing chamber. Crit Care Med 27:880-886

18. Ohashi Y, Nakai Y, Morimoto K, Tanaka A, Kakinoki Y, Ohno Y, Masamoto T, Kato A 1997 Platelet activating factor compromises airway epithelial defense functions. J Allergy Clin Immunol 100:520-526

19. Soler AP, Marano CW, Bryans M, Miller RD, Garulacan LA, Mauldin SK, Stamato TD, Mullin JM 1999 Activation of NF- $\kappa$ B is necessary for the restoration of the barrier function of an epithelium undergoing TNF- $\alpha$-induced apoptosis. Eur J Cell Biol 78:56-66

20. Xu DZ, Lu Q, Kubicka R, Deitch EA 1999 The effect of hypoxia/reoxygenation on the cellular function of intestinal epithelial cells. J Trauma 46:280-285

21. Schmitz H, Fromm M, Bentzel CJ, Scholz P, Detjen K, Mankertz J, Bode H, Epple HJ, Riecken EO, Schulzke JD 1999 Tumor necrosis factor- $\alpha$ (TNF $\alpha)$ regulates the epithelial barrier in the human intestinal cell line HT-29/B6. J Cell Sci 112:137-146

22. Inagaki-Ohara K, Yada S, Takamura N, Reaves M, Yu X, Liu E, Rooney I, Nicholas S, Castro A, Ware CF, Green DR, Lin T 2001 p53-dependent radiation-induced cryp intestinal epithelial cells apoptosis is mediated in part through TNF-TNFR1 system. Oncogene 20:812-818

23. Xu DZ, Lu Q, Swank GM, Deitch EA 1996 Effect of heat shock and endotoxin stress on enterocyte viability apoptosis and function varies based on whether the cells are exposed to heat shock or endotoxin first. Arch Surg 131:1222-1228

24. Coopersmith CM, O'Donnell D, Gordon JI 1999 Bcl-2 inhibits ischemia-reperfusioninduced apoptosis in the intestinal epithelium of transgenic mice. Am J Physio 276:G677-G686

25. Ford H, Watkins S, Reblock K, Rowe M 1997 The role of inflammatory cytokines and nitric oxide in the pathogenesis of necrotizing enterocolitis. J Pediatr Surg 32:275-282
26. Qi BQ, Williams A, Beasley S, Frizelle F 2000 Clarification of the process of separation of the cloaca into rectum and urogenital sinus in the rat embryo. J Pediatr Surg 35:1810-1816

27. Vachon PH, Cardin E, Harnois C, Reed JC, Vezina A 2000 Early establishment of epithelial apoptosis in the developing human small intestine. Int J Dev Biol 44:891898

28. Farber A, Connors JP, Friedlander RM, Wagner RJ, Powell RJ, Cronenwett JL 1999 A specific inhibitor of apoptosis decreases tissue injury after intestinal ischemiareperfusion in mice. J Vasc Surg 30:752-760

29. Kim JM, Eckmann L, Savidge TC, Lowe DC, Witthoft T, Kagnoff MF 1998 Apoptosis of human intestinal epithelial cells after bacterial invasion. J Clin Invest 102:1815-1823

30. Ikeda H, Suzuki Y, Suzuki M, Koike M, Tamura J, Tong J, Nomura M, Itoh G 1998 Apoptosis is a major mode of cell death caused by ischaemia and ischaemia/ reperfusion injury to the rat intestinal epithelium. Gut 42:530-537

31. Sandoval M, Liu X, Mannick EE, Clark DA, Miller MJ 1997 Peroxynitrite-induced apoptosis in human intestinal epithelial cells is attenuated by mesalamine. Gastroenterology 113:1480-1488

32. Allen S, Kumar M, Arnfeh B, Chester A, Dzimiri N, Duran CM, Yacoub M 1996 Influence of cooling on mesenteric vascular reactivity. Eur J Cardiothorac Surg 10:1015-1020

33. Fatica C, Gordon S, Mossad E, McHugh M, Mee R 2000 A cluster of necrotizing enterocolitis in term infants undergoing open heart surgery. Am J Infect Contro 28:130-132

34. Yamamoto R, Takasaki K, Nickols GA 1992 Purinergic vasoconstrictor component revealed by moderate cooling in the isolated mesenteric vasculature of SpragueDawley rats. J Pharmacol Exp Ther 262:1133-1138

35. Barlow B, Santulli TV 1975 Importance of multiple episodes of hypoxia or cold stress on the development of enterocolitis in an animal model. Surgery 77:687-690

36. Caplan MS, Hedlund E, Adler L, Hsueh W 1994 Role of asphyxia and feeding in a neonatal rat model of necrotizing enterocolitis. Pediatr Pathol 14:1017-1028

37. Shah U, Walker WA 2000 Adverse host responses to bacterial toxins in human infants. J Nutr 130:420S-425S

38. Lucas A, Cole TJ 1990 Breast milk and neonatal necrotising enterocolitis. Lancet 336:1519-1523

39. Caplan MS, Sun XM, Hseuh W, Hageman JR 1990 Role of platelet activating factor and tumor necrosis factor- $\alpha$ in neonatal necrotizing enterocolitis. J Pediatr 116:960 964

40. Caplan MS, Kelly A, Hsueh W 1992 Endotoxin and hypoxia-induced intestinal necrosis in rats: the role of platelet activating factor. Pediatr Res 31:428-434

41. Langer JC, Sohal SS, Mumford DA 1993 Mucosal permeability in the immature rat intestine: effects of ischemia-reperfusion, cold stress, hypoxia, and drugs. J Pediat Surg 28:1380-1383; discussion 1384-1385

42. Miller DK 1997 The role of the caspase family of cysteine proteases in apoptosis Semin Immunol 9:35-49

43. Okuno S, Shimizu S, Ito T, Nomura M, Hamada E, Tsujimoto Y, Matsuda H 1998 Bcl-2 prevents caspase-independent cell death. J Biol Chem 273:34272-34277

44. Grasl-Kraupp B, Ruttkay-Nedecky B, Koudelka H, Bukowska K, Bursch W, SchulteHermann R 1995 In situ detection of fragmented DNA (TUNEL assay) fails to discriminate among apoptosis, necrosis, and autolytic cell death: a cautionary note. Hepatology 21:1465-1468

45. Thompson CB 1995 Apoptosis in the pathogenesis and treatment of disease. Science 267:1456-1462

46. Henderson S, Rowe M, Gregory C, Croom-Carter D, Wang F, Longnecker R, Kieff E, Rickinson A 1991 Induction of bcl-2 expression by Epstein-Barr virus latent membrane protein 1 protects infected B cells from programmed cell death. Cell 65:1107-1115

47. Hartley SB, Cooke MP, Fulcher DA, Harris AW, Cory S, Basten A, Goodnow CC 1993 Elimination of self-reactive B lymphocytes proceeds in two stages: arrested development and cell death. Cell 72:325-335 